\title{
WATCH-OVER HMI for Vulnerable Road Users' Protection
}

\author{
Katrin Meinken ${ }^{1}$, Roberto Montanari ${ }^{2}$, Mark Fowkes $^{3}$, and Anny Mousadakou ${ }^{4}$ \\ ${ }^{1}$ University of Stuttgart \\ Institute for Human Factors and Technology Management \\ Nobelstr. 12, 70569 Stuttgart, Germany

\begin{abstract}
WATCH-OVER is a European project, aiming at the enhancement of road safety and the impairment of traffic accidents involving vulnerable road users (VRUs), such as pedestrians, bicyclists and motorcyclists, in urban and extra-urban areas. The project carries out research and development activities, in order to design an integrated cooperative system for accident prevention. In this paper, the concept of the Human Machine Interface of the WATCH-OVER system is discussed and its user-centred approach, based on a user requirement survey, is described. Regarding the HMI, the basic functionalities and elements, as well as the preliminary guidelines that endorse the WATCH-OVER system approach, are presented.
\end{abstract}

Keywords: Vulnerable road users, HMI, road safety, Powered Two-Wheeler's.

\section{Introduction}

Throughout the last 30 years, the overall volume of road traffic has tripled (European Commission, 2003). Even though the number of road deaths has fallen by half in these years, it is still at an unacceptable high number. New on-board information and communication technologies offer considerable potential for reducing the number of traffic accident victims. The WATCH-OVER project aims at avoiding road accidents that involve VRUs, namely pedestrians, bicyclists and motorcyclists, by developing an integrated cooperative system for accident prevention. This topic is in line with the

C. Stephanidis (Ed.): Universal Access in HCI, Part II, HCII 2007, LNCS 4555, pp. 488-496, 2007.

(c) Springer-Verlag Berlin Heidelberg 2007 
ambitious target of the EC, to reduce the total number of road fatalities by $50 \%$ by 2010.

WATCH-OVER is a specific targeted project, co-funded by the European Commission Information Society Technologies (IST), that started its activities in January 2006. The core of the system, based on sensor and communication technologies, is the interaction of an on-board module and a user module, in order to cover a wide traffic scenario, including blind spots. Current sensor technologies cannot "see" behind obstacles and have a limited view of lateral and longitudinal areas. The main difficulty in detecting VRUs is therefore the limited "visibility" of car drivers and of in-vehicle sensor based systems. WATCH-OVER aims at improving these soft spots, by combining the most promising communication technologies with the most promising sensor technologies. Besides focussing on the exploitation of these advanced communication and sensing technologies, the main focus of the project is the design and development of the human machine interface (HMI) for both the driver and the vulnerable road user. Therefore, different solutions for the HMI have to be found: One would be an in-vehicle device, whereas the others would be based upon wearable devices for pedestrians, bicyclists and motorcyclists.

To set up an effective HMI, which meets the demands of the future users, it is essential to consult existing guidelines and specifications on the one hand, and to carry out a thorough examination of user requirements and identify specific traffic scenarios, in which the system is considered as valuable, on the other hand. The present paper describes the user-centred development procedure, based on general guidelines and regulations and on the results of a conducted user needs survey, concerning HMI, including the requirements of an effective warning strategy.

\section{System Architecture}

The WATCH-OVER project focuses its activities on the design and development of an integrated cooperative vehicle-user system, for the prevention of accidents involving VRUs, in urban and extra-urban scenarios. The system architecture is based upon the cooperation of innovative wireless short range communication technologies and vision sensor technologies. This cooperative platform shall extend the actual coverage of the state of the art technologies and will be open to integrate localisation technologies. Therefore, the following specifications are considered as important and will be integrated in the design of the WATCH-OVER system:

- The extension of the "protection concept" by an effective warning and/or intervention strategy.

- Increased vehicle speed range (up to $50 \mathrm{~km} / \mathrm{h}$ ), at which the system is operable.

- A high reliability of signal recognition in each scenario.

- Timely performance of the detection and localisation of VRUs.

- Low cost and low power consumption of components. 
- Increased processing speed (more than $10 \mathrm{~Hz}$ ).

- Increased sensor coverage (0-20m).

The key challenge in implementing these attitudes into the system architecture is to cover the various urban and extra-urban traffic scenarios, involving a variety of VRUs, in very different conditions. Thus, the in-vehicle module of the WATCHOVER system is intended to enable the following functionalities:

- Real-time detection of pedestrians, bicyclists and motorcyclists, equipped with the WATCH-OVER module.

- Calculation of the relative positioning of the vehicle vs. VRU (relative motion analysis).

- Detection of dangerous situations.

- Appropriate warning of the driver, providing information only in really dangerous situations.

The vulnerable road user module on the other hand is conceived to:

- Promptly answer to vehicle's stimulus, delivering its identification parameters.

- Send back self-localisation parameters.

- Give feedback to the VRU with an appropriate HMI.

The modules for the VRUs are designated to be integrated into wearable objects, like helmets, shoes, watches, rucksacks or consumer electronics.

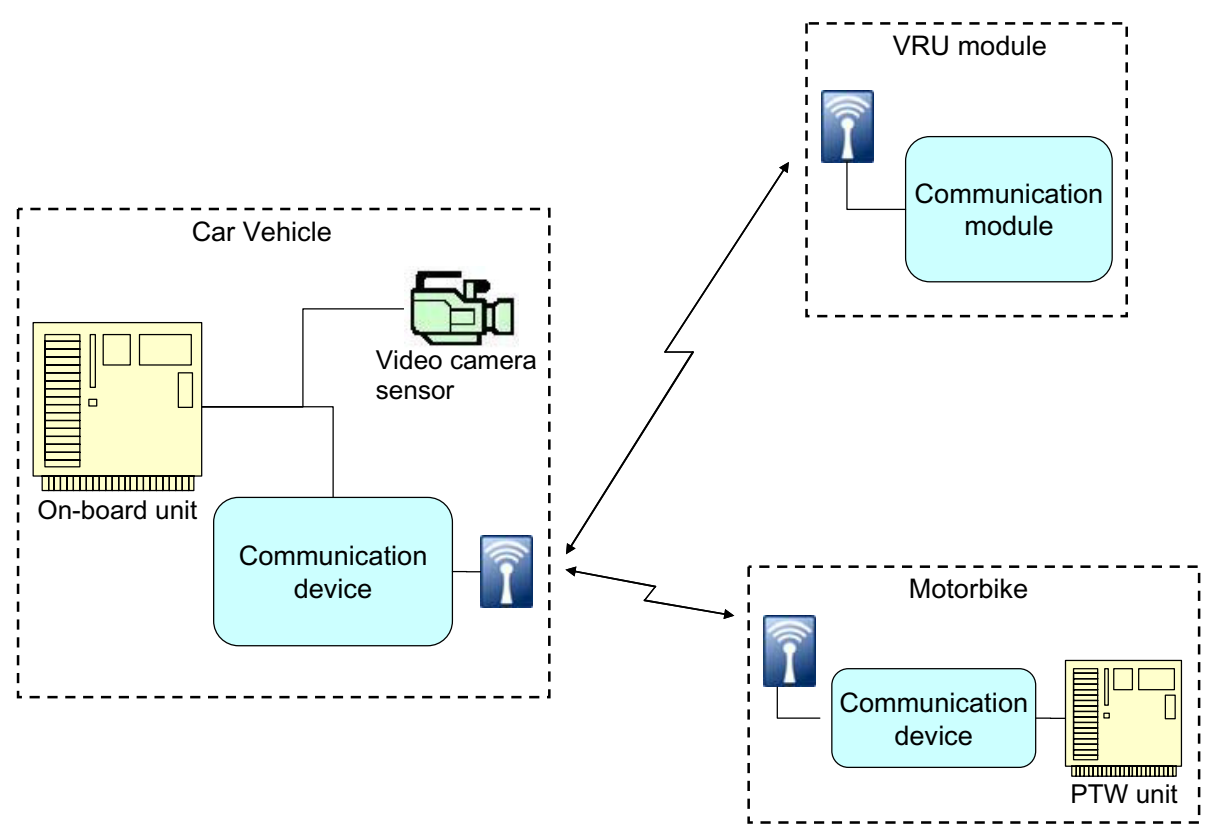

Fig. 1. WATCH-OVER system architecture and components 


\section{HMI Concept and Warning Strategy}

To derive the concept for the HMI of the WATCH-OVER system, different sources had to be considered. Not only general guidelines for HMI design and common requirements for warning strategies were consolidated but also the specific user requirements, related to the WATCH-OVER topics, were examined.

\subsection{Appliance of Preliminary Guidelines}

General guidelines for in-vehicle systems were used, together with specific guidelines for the design of warning devices, to derive the basic HMI and warning concept. In 2001 the European Commission concluded a process, which started on 1999, for the development of a set of guidelines for the design of safety on-board information systems. This document is named "European Statement of Principles on Human Machine Interface (HMI) for In-Vehicle Information and Communication Systems" (ESoP) and was updated in December 2006. Considering that the driver's primary task is the safe control of the vehicle in a complex and dynamic traffic environment, the primary goal of the principles is to fulfil the requirements. All contemplated guidelines summarise essential safety aspects, to be considered for the HMI for in-vehicle information and communication systems. These guidelines apply to all components and aspects of a system that the driver will interact with while driving. The most important declarations need to be included in the WATCH-OVER system, such as that:

- The system must comply with relevant regulation and standards.

- The system supports the driver and does not increase driver distraction from driving task.

- The system shall not require uninterruptible sequences of interaction.

- The system does not distract or visually entertain the driver.

- No part of the system should obstruct the driver's view of the road scene.

- The system response (e.g. feedback, confirmation) following driver input should be timely and clearly perceptible.

- Information which has the highest safety relevance should be given priority.

- The behaviour of the system should not adversely interfere with the display or controls required for the primary driving task and for road safety.

Thus, the main goal of the WATCH-OVER HMI must be to avoid driver confusion and overload. Only information that will assist the driver more than distract him/her in complex traffic scenarios in urban and extra-urban areas should be provided by the HMI.

\subsection{Warning Strategy}

The WATCH-OVER warning strategy follows the approach of Wickens et al. (2004), as they state in their work that "the goal [of a warning] is to get the user to comply with the warning and, therefore, use the product in a safe way, or avoid unsafe behaviour." In order to ensure a secure handling of the future system, four elementary requirements have to be attended to: 
- The warning must be noticed.

- The warning must be perceived (read/heard).

- The warning must be understood.

- The warning must be accepted.

By applying these criteria, an efficient design of a warning strategy will first of all draw the attention of the driver or the vulnerable road user. Then, it has to be assured that the warning message is not only physically perceived, but, moreover, cognitively understood and then accepted by the driver or the vulnerable road user. The system has also to give information about the identified risk and about possible and recommendable actions, in case they are not obvious.

\subsection{User Requirements}

The definition of user requirements is an essential step in designing a system, intended to effectively serve the needs of the future user. The user requirements have to be considered since the beginning of the project and are of immediate importance for the development and implementation of the system's hardware and software architecture. In WATCH-OVER, user requirements have been defined through 154 questionnaires, answered by non-technical experts from 9 countries (Austria, Chech Republic, France, Germany, Greece, Italy, Poland, Switzerland, the Netherlands), who were pedestrians and drivers themselves and commute regularly with cars, motorcycles and bicycles. According to the answers given to HMI related questions, the following HMI requirements arise:

\section{In case of no accident risk}

1. the system should only inform the driver of the presence of VRUs (location, distance, etc.) after explicit demand;

2. the visual information should appear on the head up display or on the instrument cluster;

3. the system should inform the driver of the presence of VRUs, regarding the distance and the heading of the vulnerable road user on demand only.

\section{In case of an acute accident risk due to the presence of a VRU}

4. the system should warn the driver;

5. the warning should be a tone/beep or an icon on the display;

6. secondary important information provided includes the relative position, the weather, the height of the pedestrian and the momentum;

7. these information (see item 5 and 6) should be presented by an icon on display or by a tone/beep.

Also, previous projects demonstrate that driver assistance systems designed to prevent accidents with VRUs are highly attractive. Users' assessments on different HMI's of dissimilar systems show that following conclusions should be considered important in HMI development and therefore for the development of the WATCHOVER system as well: 
- The allowance for false alarm should be very low.

- If a driver perceives too many false alarms, the warning will be ignored (Lee, See 2004).

- The warning should be given acoustically and therefore must be heard.

- Mere visual information may not be noticed in-time, and might possibly decrease safety due to its distraction effect. A combination with visual information might be useful, because of redundancy.

- The warning should be given with adequate timing, in order to allow the driver to react well considered.

\subsection{WATCH-OVER HMI Concept}

In the course of the identification of the user requirements, an expert's workshop has been held, in order to discuss the implementation of the WATCH-OVER HMI. One main objective was to find an approach that limits the number of false alarms or even completely avoids them and to evade issues of an information overload. Therefore, it is crucial to be coherent throughout the warning strategy and by this avoid redundancy. The WATCH-OVER HMI concept provides a continuous evaluation of the risk level by the system. If the risk level is approaching a prior specified threshold, the system will warn the driver or the VRU respectively. The thresholds will be identified according to the different risk levels.

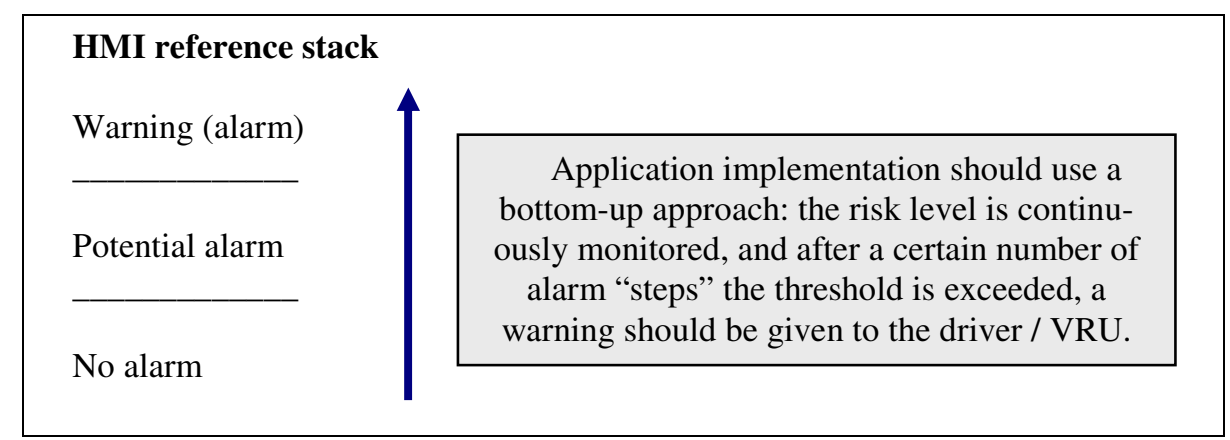

Fig. 2. HMI approach followed within the WATCH-OVER project

The above discussed concept is introduced, in order to avoid the development of a system, that could potentially distract the driver or the VRU, instead of assisting in already complex traffic situations. If the HMI interface is too pervasive, with an intensive visual or acoustical output presented to the user, it may lead to switching the system off altogether. Therefore, it is necessary to eliminate false alarms, as well as an overload of information in all cases, if possible.

Through the HMI, the communication with the user will be established. Thus, the HMI constitutes the physical representation of the warning strategy. It is important that the HMI set-up is adaptable to both the personal preferences of the user, as well as to his/her cultural background, in order to guarantee a good acceptance. That means, e.g. that the driver or the VRU should have control of the loudness of auditory information, due to the likelihood of distraction or of a noisy surrounding. Or that, 
concerning the exploitation of the international market, internationally and/or nationally agreed standards, related to icons or symbols, should be used and speech output has to be at least in a language that the user is able to understand.

\subsubsection{Special Requirements for the HMI for VRUs: Powered Two-Wheelers}

Both the ESoP and other guidelines concern mainly the automotive sector. The research in the domain of Powered Two-Wheeler's (PTWs) is rather limited and most studies concerning the safety aspect do not deal with information systems at all. However, the neighbouring area of automotive research is well explored and the guidelines for this area may be used to some extent for PTWs, although some modifications are necessary.

There are some important differences between the PTW domain and the automotive domain. The PTW-driver is, for instance, much more affected by rain, wind and noise, since a standard PTW is not sheltered. This means that the design of a PTW information system has to take into consideration such things as dirty or wet displays or helmet visor and high noise levels, due to rain, wind, high speed or city traffic. Hence, the automotive design guidelines have to be utilised in a very conservative manner in the PTW-domain. The recommended limit should always be used rather than the legal limit: for instance the font size on displays could be augmented, in comparison to the minimum accepted size and the sound level for warning signals and other information should be adjustable to the noise environment inside a helmet.

The automotive guidelines may hence represent an important help in designing for PTWs, but every decision has to be made with keeping in mind the vital differences between the PTW and the automotive driving conditions.

\subsubsection{Special Requirements for the HMI for VRUs: Pedestrians and Bicyclists}

There is even less guidance on the design of an HMI for the pedestrian and bicyclist VRUs than there is for the PTW.

For the bicyclists, there are however some environmental considerations, that are somewhat similar to those noted above for the PTW. The exposed nature of the environment for the bicyclist also requires careful consideration, with regard to implementation of any HMI for this environment. The range of characteristics of the bicycle riders may also be somewhat different than for the PTW riders, with no restriction on age, due to the lack of licensing requirements, for example. There are also practical considerations with regard to HMI packaging and implementation for the bicyclist without the availability of any regular power sources and other electrical equipment and displays, that would be available on the PTW on the majority of most bicycles. An HMI that is self-powered and potentially worn by the user should be considered.

In this respect, this may have similar requirements to the HMI for a pedestrian who has, additionally, even less constraints in terms of legal requirements, licensing and power availability, than the bicyclist.

In both cases, HMI that can deliver readily understood warning requirements to the user, in a clear and unambiguous manner, must be sought. This must consider all potential sensory modalities, that could be realised in such an operational setting. These, potentially, must be understandable to users across a wider range of age, than that for the automotive and PTW users. 


\section{Conclusions and Outlook}

The WATCH-OVER European project aims at designing and developing an integrated cooperative system for the prevention of accidents, involving VRUs in urban and extra-urban areas. The project is co-funded by the European Commission and is supported by EUCAR. The project Consortium is composed of 13 project partners from six different European countries, presenting vehicle and PTW manufacturers, automotive suppliers, technology and research centres for the development and testing phase.

The system is based on the cooperation of communication and sensor technologies and will provide users with an in-vehicle module, to warn drivers of approaching VRUs on the one hand and wearable modules, that call attention of VRUs to critical traffic situations, on the other hand. One major challenge in the development process is the design of an efficient HMI, that is of assistance in dangerous scenarios and does not distract the driver's or vulnerable road user's attention from the driving task or the surrounding traffic.

The development phase of the WATCH-OVER HMI has just started and will therefore be further addressed and investigated within the ongoing project activities. Executable prototypes of the HMI are planned to be tested and validated with driving simulators. The cooperative platform of different modules - in-vehicle and VRUsided - will significantly help to further enhance road safety and to accomplish the goal of reducing the number of road fatalities.

Acknowledgements. We wish to acknowledge to the WATCH-OVER project Consortium of the PReVENT IP project, for their valuable contributions to this work. The WATCH-OVER project is partially funded by the EC.

\section{References}

1. Andreone, L., Bekiaris, E.A., Guarise, A., Mousadakou, A. (2006), D2.1 Requirements and use cases, WATCH-OVER project, (July 2006)

2. Andreone, L., Guarise, A., Lilli, F., Gavrila, D.M., Pieve, M. (2006), Cooperative Systems for Vulnerable Road Users: The Concept of the WATCH-OVER Project, 13th ITS World Congress, London (2006)

3. Barber, C.: Psychological aspects of conventional in-car warning devices. In: Stanton N (Ed.), Human Factors in Alarm Design, pp. 193-205. London: Taylor \& Francis (1994)

4. European Commission, Commission Recommendation of 21 December 2005 on safe and efficient in-vehicle information and communication systems: A European statement of principles on human machine interface (2006)

5. Hagenmeyer, L., Marberger, C., Bekiaris, E., Nikolaou, S.: Preliminary Guidelines for the Personalization of the HMI of Hypovigilance-Management-Systems. Conference Proceedings HCI (2005)

6. European Commission; Communication from the Commission - European road safety action programme - Halving the number of road accident victims in the European Union by 2010: a shared responsibility COM/2003/0311 final

7. ISO, 8: Road vehicles - Ergonomic aspects of transport information and control systems Specifications and compliance procedures for in-vehicle visual presentation (1500) 
8. Lee, J.D., See, K.A.: Trust in Automation: Designing for Appropriate Reliance, Human Factors, vol. 46(1)

9. Lerner, N.D., Kotwal, B.M., Lyons, R.D., Gardner-Bonneau, D.J.: Preliminary Human Factors Guidelines for Crash Avoidance Warning Devices. National Technical Information Service, Springfield (1996)

10. Marberger, C., Dangelmaier, M., Bekiaris, E., Nikolaou, S. (2004). User centred HMI development for the AWAKE vigilance monitoring system. In: Conference Proceedings FISITA (2004)

11. Oltersdorf, K.M., Mayer, B., Maximov, K. (2003), D02.3 User Needs Investigation Results, PROTECTOR project (February 2003)

12. WATCH-OVER web site: http://www.watchover-eu.org

13. Wickens, C.D., Lee, J.D., Gordon Becker, S.E., Liu, Y.: An introduction to human factors engineering. Pearson Education, Upper Saddle River, New Jersey (2004) 